\title{
Estudo de Validação do Questionário de Coping Conjugal Prospetivo e do seu Valor Preditivo no Bem-estar Pessoal e no Ajustamento Diádico
}

\author{
Validation Study of the Prospective Conjugal Coping Questionnaire and its \\ Predictive Value in Personal Well-being and Dyadic Adjustment
}

\author{
Susana Costa-Ramalho ${ }^{1}$, Alexandra Marques-Pinto ${ }^{2}$ e Maria Teresa Ribeiro ${ }^{2}$
}

\begin{abstract}
Resumo
Este estudo propôs-se examinar algumas propriedades psicométricas do Questionário de Coping Conjugal Prospetivo (QCCP), designadamente a validade preditiva do coping prospetivo na explicação do bem-estar pessoal e ajustamento diádico. Um total de 451 adultos numa relação conjugal respondeu ao QCCP, ao Questionário de Bem-Estar Pessoal (Keyes et al., 2008) e à Escala de Ajustamento Diádico (Spanier, 1976). Um modelo unifatorial de coping prospetivo com seis itens mostrou um melhor ajustamento aos dados do que o modelo bifatorial original. Análises de regressão múltipla evidenciaram que as estratégias de coping prospetivo explicam uma percentagem significativa de variância em todas as dimensões de ajustamento diádico e bem-estar pessoal. Os resultados encorajam a prosseguir o estudo deste instrumento no âmbito da conjugalidade, a fim de disponibilizarmos um instrumento válido e útil para a avaliação de como os cônjuges assumem um papel ativo na construção e manutenção da sua relação de casal.
\end{abstract}

Palavras-chave: Questionário de Coping Conjugal Prospetivo, validação, conjugalidade, bem-estar, ajustamento diádico

\begin{abstract}
This study set out to examine some psychometric properties of the Prospective Conjugal Coping Questionnaire (QCCP), namely the predictive value of the prospective coping in the explanation of personal well-being and dyadic adjustment. A sample of 451 adults in a couple relationship answered the QCCP, the Mental Health Continuum-SF (Keyes et al., 2008) and the Dyadic Adjustment Scale (Spanier, 1976). A six-items unifactorial prospective coping model showed a better fit to the data than the original two-factor solution. Multiple regression analyses showed that the prospective coping strategies explained a significant proportion of variance in all dimensions of dyadic adjustment and personal wellbeing. The results encourage us to pursue the study of this instrument within the scope of couplehood, in order to provide a valid and useful tool for the assessment of how people take an active role in building and maintaining the marital relationship.
\end{abstract}

Keywords: Prospective Conjugal Coping Questionnaire, validation, couplehood, well-being, dyadic adjustment

Este Estudo foi financiado por uma Bolsa de Doutoramento atribuída ao primeiro autor pela Fundação para a Ciência e Tecnologia (SFRH/BD/70789/2010).

\footnotetext{
${ }^{1}$ Faculdade de Psicologia da Universidade de Lisboa e Faculdade de Psicologia e de Ciências da Educação, Universidade de Coimbra, Portugal. Telefone: 0035196684734. E-mail: susana.costaramalho@gmail.com

${ }^{2}$ Faculdade de Psicologia da Universidade de Lisboa, Portugal.
} 


\section{Introdução}

O conceito de coping proativo, globalmente definido enquanto foco pessoal na definição e concretização dos objetivos (Schwarzer \& Taubert, 2002), tem-se revelado como um preditor significativo do bem-estar individual (e.g. Sohl \& Moyer, 2009; Uskul \& Greenglass, 2005) e do ajustamento em contextos de vida relevantes (e.g. ajustamento académico; Gan, Yang, Zhou, Hu, \& Zhang, 2007). Não se conhecem, contudo, estudos publicados que tenham avaliado o papel desta dimensão do coping no âmbito da vida conjugal, sendo esse um dos objetivos centrais do presente estudo.

Revendo a literatura no âmbito das relações amorosas, a dimensão facialmente mais próxima do coping proactivo parece ser o conceito de autorregulação relacional (relationship selfregulation, no original). Este foi inicialmente introduzido por Halford, Sanders e Beherns (1994) e aprofundado posteriormente por Halford, Wilson, Lizzio e Moore (2002) para designar o conjunto de estratégias que cada indivíduo usa por sua iniciativa para concretizar a construção da sua relação amorosa, assim como a sua persistência em implementá-las. Muito embora a autorregulação seja em si mesma um processo individual, a investigação tem documentado o seu impacto nas relações interpessoais e, concretamente, a sua influência na manutenção de relações positivas e saudáveis (e.g. Halford et al., 1994).

A crença central de que os indivíduos são capazes de regular o seu comportamento, com origem na teoria da autorregulação individual (e.g. Bandura, 2001), pode assim ser aplicada no contexto das relações de casal onde é igualmente entendida como um ciclo ou processo individual de avaliação, definição de objetivos, implementação da mudança e persistência nos esforços de mudança. Relativamente a este conceito, os programas de educação ou enriquecimento conjugal bem como de terapia de casal têm vindo progressivamente a enfatizar esta perspetiva de autorregulação como o melhor caminho para a mudança sistémica nas relações de casal (e.g. Markman, Stanley, \& Blumberg, 2010).

Por outro lado, diversos estudos desenvolvidos na última década têm colocado em evidência que: (a) a autorregulação se correlaciona positivamente e atua como um preditor significativo do sucesso e satisfação relacional (Halford, Lizzio, Wilson, \& Occhipinti, 2007; Shafer, Jensen, \& Larson, 2012), explicando entre 25-33\% da variância na satisfação; (b) o empenho dos cônjuges na consecução de objetivos específicos para melhorar a sua relação - que conceptualmente se aproxima do que poderíamos designar de coping proactivo ao serviço do sucesso conjugal - é um caminho importante para níveis significativamente superiores de satisfação relacional (Halford et al., 2007).

$\mathrm{Na}$ ausência de estudos anteriores, enquadramos assim o nosso objeto de estudo - as estratégias de coping orientado para o futuro (Gan, et al., 2007; Schwarzer \& Taubert, 2002) no âmbito dos designados mecanismos de autorregulação individual (cf. Halford et al., 2002: "conjunto de estratégias que cada indivíduo usa por sua iniciativa para concretizar a construção da sua relação amorosa"), pretendendo explorar-se a sua validade no contexto das relações amorosas. Ser capaz de se preparar para gerir potenciais stressores, ao mesmo tempo que se constroem recursos gerais que facilitam o alcance de objetivos e a promoção do crescimento pessoal, parece ser um fator relevante para o bem-estar do casal. No presente estudo, definimos assim como objetivos centrais validar uma medida de coping prospetivo ou orientado para o futuro - entendido como uma dimensão de autorregulação relacional - no contexto da relação amorosa de indivíduos portugueses e explorar o seu papel preditor no bem-estar pessoal e no ajustamento diádico.

\section{Dos conceitos de coping proativo e preventivo ao de coping orientado para o futuro}

O conceito de coping proactivo originalmente definido por Greenglass, Schwarzer, Jakubiec, Fiksenbaum, e Taubert (1999) - pretende integrar as qualidades pessoais de gestão e de autorregulação na consecução de objetivos, potenciando a iniciativa e uma visão individual de sucesso (Schwarzer, 1999). Com o seu foco positivo, este conceito está em sintonia com as perspetivas da psicologia que incorporam o potencial humano, o esforço pessoal, os motivos e as capacidades dos seres humanos, mais do que 
apenas os seus limites e sofrimentos (Seligman, 1990). Os autores definiram o coping proativo como sendo guiado pelas crenças no potencial de mudança que cada pessoa considera ter para incrementar em si e no seu contexto mudanças positivas. Na perspetiva de um indivíduo proativo, o sucesso depende da responsabilidade que ele próprio assume sobre os resultados.

O conceito de coping proactivo difere das formas tradicionais (e mais estudadas) de coping em vários aspetos (Greenglass, 2002b). Por um lado, enquanto estas tendem a ser reativas, na medida em que lidam com acontecimentos stressantes já ocorridos, o coping proativo é dirigido para o futuro e visa a acumulação de recursos que promovam o crescimento pessoal e a consecução de objetivos. A segunda distinção prende-se com o facto de que o coping reativo se relaciona com a gestão de riscos enquanto o proactivo se centra na gestão de objetivos (Schwarzer, 1999). Os riscos, desafios e oportunidades do futuro não são considerados como perigos ou ameaças, mas antes como desafios a ultrapassar. Em terceiro lugar, a motivação para o coping proativo é mais positiva do que nas formas tradicionais de coping, uma vez que ela deriva da perceção das situações como estimulantes e desafiantes, não como ameaças.

Greenglass et al. (1999) introduziram em simultâneo o conceito de coping preventivo, distinto do anterior pela motivação subjacente. Este relaciona-se com a antecipação de potenciais ameaças ou stressores - antes da sua ocorrência com base na experiência ou conhecimento pessoal. Segundo Schwarzer (2000), o coping preventivo tem como finalidade aumentar os recursos pessoais de modo a diminuir a probabilidade de que surjam os acontecimentos stressores temidos e reduzir a severidade das consequências do stress, caso ocorra. Nesta medida distingue-se do coping proactivo o qual, como vimos, não se baseia em ameaças mas é antes impulsionado pela avaliação do desafio e pela consecução de objetivos.

Na prática, ambos os tipos de coping - proativo e preventivo - podem dizer-se dirigidos para o futuro - por oposição a reativos face a acontecimentos passados (Greenglass, 2000b), manifestando-se em parte no mesmo tipo de comportamentos, como sejam o desenvolvimento de competências pessoais, a acumulação de recursos e o planeamento. Esta proximidade conceptual tem dado origem a algumas incorreções na literatura, nomeadamente com alguns autores a considerar que os comportamentos realizados com antecedência para evitar potenciais stressores podem ser considerados como proativos (e.g. Aspinwal \& Taylor, 1997). Mais recentemente, Gan et al. (2007) sugeriram a utilização do termo "coping orientado para o futuro" para incluir ambos os tipos de coping, o que nos parece apropriado visto serem ambos (apesar das suas diferentes motivações e conteúdos) dirigidos para o futuro. Adoptamos pois, por ora em diante, a designação de coping prospetivo para referirmos o conjunto das estratégias de coping, orientadas para o futuro, de tipo proativo $\mathrm{e}$ preventivo.

Dos resultados que anteriormente referimos podem derivar múltiplas possibilidades para investigações sobre os processos de coping que parecem, em simultâneo, diminuir a perturbação e promover o bem-estar (Greenglass, 2002a). Mais especificamente, se considerarmos que o contexto das relações de casal é necessariamente (não apenas, mas também) revestido de desafios e de stressores, o coping será certamente uma dimensão importante a estudar, não existindo, tanto quanto é do nosso conhecimento, estudos publicados sobre o papel do coping prospetivo no contexto das relações de casal, razão pela qual desenvolvemos este estudo.

\section{Do Inventário de Coping Proativo ao estudo atual}

O designado Inventário de Coping Proativo (PCI) foi desenvolvido por Greenglass et al. (1999) com base na Teoria de Coping Proativo de Schwarzer (1999) que distingue entre coping reativo, antecipatório, preventivo e proativo. $\mathrm{O}$ seu objetivo era possibilitar a avaliação de diferentes dimensões do coping usadas pelos indivíduos em situações de stress ou perante a sua antecipação. Deste inventário faziam parte sete subescalas, com índices de consistência interna variando entre .80 e .85 : (a) coping proactivo (14 itens); (b) coping refletivo (11 itens); (c) planeamento estratégico (4 itens); (d) coping preventivo (10 itens); (e) procura de suporte instrumental (8 itens); (f) procura de suporte 
emocional (5 itens); e (g) evitamento. Na linha do anteriormente referido, as subescalas de coping proactivo e preventivo são as duas que consideramos "orientadas para o futuro".

Dos estudos já realizados com o PCI, salientamse como resultados interessantes a correlação positiva do coping proativo com a satisfação com a vida (Uskul \& Greenglass, 2005) e com a eficácia profissional (Greenglass, 2005); a correlação negativa com a depressão (e.g. Pasikowski, Sek, Greenglass, \& Taubert, 2002), auto-culpabilização, negação e burnout (e.g. Greenglass, Stokes, \& Fiksenbaum, 2005). Outros estudos enfatizaram o seu papel preditor nas dimensões de satisfação com a vida e engagement (e.g. Gan et al., 2007). No caso do coping preventivo, os resultados têm sido menos evidentes (Aspinwall \& Taylor, 1997), sugerindo que tem também um impacto positivo no ajustamento individual (e.g. Gan et al., 2007) mas os seus efeitos mediadores parecem ser inferiores aos do coping proactivo.

Segundo Schwarzer (comunicação pessoal, 5 de Maio, 2011), seria pertinente compreender o papel do coping preventivo e proativo no contexto das relações de casal. Aspinwall (2005) sugeriu também que se explorasse o impacto desta dimensão prospetiva nos relacionamentos conjugais. Concretamente, de acordo com Schwarzer, seria relevante partir da escala PCI e desenvolver a sua adaptação ao contexto diádico, nomeadamente:

(a) Explorar a multidimensionalidade (Endler \& Parker, 1994) do construto de coping nas suas vertentes preventiva e proactiva, no âmbito da conjugalidade;

(b) Adaptar os itens mais relevantes das duas subescalas para o contexto específico da relação de casal: porque as estratégias de coping não ocorrem num vácuo social (Greenglass, 2002b), sendo hoje pertinente levar em consideração o contexto social (neste caso, o contexto conjugal) em que as estratégias de coping intervêm;

(c) Explorar as relações entre as estratégias de coping e resultados de bem-estar, individuais e/ou conjugais, por se considerar que a função do coping pode não ser apenas a de aliviar o distress mas também a de aumentar o potencial humano de crescimento, satisfação e qualidade de vida (Greenglass et al., 1999).

\section{O nosso estudo}

Assumindo a perspetiva de que o entendimento das estratégias de coping enquanto recurso de crescimento pessoal e de concretização de objetivos possibilita uma visão mais compreensiva da vida humana (Schwarzer \& Taubert, 2002), o presente estudo visa explorar os comportamentos de coping num contexto particularmente desafiante e potencialmente stressante como é o das relações de casal. Concretamente, tem por primeiro objetivo adaptar ao contexto conjugal e numa amostra da população portuguesa as subescalas proativa e preventiva do Inventário de Coping Proativo (Greenglass et al., 1999). Nesse sentido, com base numa amostra de adultos casados ou em união de facto há menos de 11 anos (critério de inclusão), será estudada a estrutura fatorial do instrumento, as suas qualidades psicométricas e a validade de critério concorrente. Procuraremos ainda compreender o efeito preditor do coping nos outcomes relacionais de bem-estar pessoal e de ajustamento diádico. $\mathrm{O}$ novo instrumento - que designámos de Questionário de Coping Conjugal Prospetivo (QCCP) - pretende assim alargar a compreensão do fenómeno do coping orientado para o futuro para o contexto particular das relações conjugais.

\section{Método}

\section{Participantes}

Participaram neste estudo 451 adultos $(67 \%$ mulheres) entre os 21 e os 64 anos ( $\mathrm{M}=33.5$; $\mathrm{DP}=5.34)$. Por opção metodológica, definimos como critério de inclusão o estudo de indivíduos em relações conjugais (casamentos e uniões de facto $^{1}$ ) nos primeiros dez anos de vida em comum. A justificar esta escolha, a perspetiva teórica de DeFranck-Lynch (1986 cit. in Relvas, 1996), que caracteriza como difícil o designado estádio de fusão correspondente à primeira dezena de anos da relação conjugal, teoria esta que se vê

\footnotetext{
${ }^{1}$ No caso das uniões de facto, usámos como critério a referência legal portuguesa (Lei n. ${ }^{\circ}$ 135/99): pessoas de sexo oposto que vivam em comunhão de habitação, mesa e leito há mais de dois anos mas que não tenham um vínculo de casamento.
} 
claramente reforçada pelos dados estatísticos: em Portugal, de acordo com os Censos de 2011 (INE), observa-se uma tendência para o aumento do número de divórcios em casamentos muito recentes (dos 0 aos 4 anos), que cresceram de 12.2\% em 1992 para 16.1\% em 2011. O grupo dos 5 aos 9 anos de casamento mantém-se maioritário nos divórcios por duração de casamento $(19.5 \%$ em 2011). Eram casados $66.3 \%$ e $33.7 \%$ coabitantes.

Cada participante respondeu individualmente ao estudo, sendo assim o sujeito (e não o casal) a nossa unidade de análise. A maioria $(91 \%)$ eram trabalhadores no ativo e perto de $6 \%$ desempregados. Relativamente ao nível de estudos, 35\% referiram ter menos do que formação universitária; $52.5 \%$ completaram o ensino superior e $12.4 \%$ estudos pós-graduados. Os participantes viviam com os seus cônjuges desde alguns meses até dez anos ( $M=5.94$; $S D=2.91)$. Quase dois terços dos participantes (63.9\%) tinham filhos $(M=.88 ; S D=.92)$ da atual relação conjugal.

\section{Procedimento}

O presente estudo insere-se no Projeto de Doutoramento do primeiro autor, tendo sido aprovado pelo Conselho Científico da Faculdade de Psicologia da Universidade de Lisboa, órgão que à data era responsável pela sua avaliação científica e ética. Os dados foram recolhidos entre Abril de 2012 e Julho de 2013 em dois formatos: (a) numa versão papel e lápis obtida junto de uma amostra de conveniência (61.2\% da amostra total) em contactos individuais ou de grupo (e.g. reuniões de pais em escolas/infantários); (b) numa versão online ( $38.8 \%$ da amostra) com recurso a métodos de amostragem de bola de neve (emails e redes sociais). Aos potenciais respondentes era pedido que participassem num estudo sobre os fatores positivos da conjugalidade e seus contributos para o bem-estar dos cônjuges e da relação. No primeiro caso, toda a informação relevante sobre o estudo (natureza e objetivos, contexto de investigação, investigadores, tempo médio de preenchimento, critérios de inclusão, etc.) era apresentada na primeira página que terminava com espaço para a assinatura do consentimento informado. $\mathrm{O}$ anonimato $\mathrm{e}$ confidencialidade dos dados foram sempre assegurados, sendo transmitido aos participantes que a qualquer momento poderiam - por sua vontade - abandonar o preenchimento dos questionários. No formato online, o contacto redigido para disseminação do estudo incluía uma ligação de acesso para a página de apresentação e de consentimento informado do protocolo. Este foi construído numa plataforma comercial que não registava o endereço de IP dos respondentes, ficando assim assegurado o seu anonimato. A página de consentimento informado disponibilizava a mesma informação de rosto da versão papel, esclarecendo ainda sobre a facilidade em abandonar o preenchimento digital a qualquer momento. Em ambos os casos, o participante tinha acesso a um email de contato da equipa de investigação, podendo por essa via manifestar a sua vontade em receber as conclusões do estudo.

\section{Medidas}

QCCP $\mathrm{O}$ coping conjugal prospetivo foi avaliado com base numa adaptação das subescalas do coping proactivo e do coping preventivo que integram o Inventário de Coping Proativo (Greenglass et al., 1999). A versão portuguesa deste Inventário tinha já sido validada por Alvarez, Marques-Pinto e Gregg (2010) e foi dela que partimos para a adaptação da versão conjugal (cf. Quadro 1) que designámos por Questionário de Coping Conjugal Prospetivo (QCCP). Selecionámos 4 itens de cada subescala, conservando a equivalência operacional do instrumento (Herdman, Fox-Rushby, \& Badia, 1998), na medida em que se manteve o mesmo formato, as instruções, o método de administração e as escalas de medida. O critério base para seleção foi a validade facial dos itens, sendo escolhidos os que revelavam no formato original um maior ajustamento e facilidade de apropriação ao domínio de comportamento a ser avaliado (relação conjugal). A adaptação dos itens consistiu principalmente na introdução de expressões verbais curtas (e.g. "na/da relação conjugal" ou "com o meu cônjuge") com o fim de direcionar os participantes para o contexto específico a avaliar. A nota dos participantes era obtida pela soma dos quatro itens de cada 
subescala. Conforme apresentado no Quadro 1, exemplos de itens são: "Tento determinar o que preciso fazer para que a relação conjugal tenha sucesso" (dimensão proativa) e "Previno-me para evitar situações que possam pôr em perigo a minha relação conjugal" (dimensão preventiva). No final do processo de adaptação, o instrumento foi administrado a vinte participantes a fim de verificar a adequação do vocabulário e a compreensão homogénea dos itens. Uma vez que não surgiram dúvidas e todos os participantes referiram a facilidade de preenchimento, os itens permaneceram tal como apresentados.

\section{DAS (EAM)}

O ajustamento diádico, variável relacional escolhida para verificação da validade de critério, foi medido pela Dyadic Adjustment Scale Escala de Ajustamento Mútuo (Spanier, 1976; versão portuguesa Lourenço \& Relvas, 2003). É composta por 32 itens que avaliam diversos aspetos do funcionamento conjugal, com pontuações mais elevadas a indicarem melhor ajustamento. Produz um resultado global de ajustamento diádico $(\alpha=.91)$ e resultados em quatro subescalas: Consenso (13 itens, e.g. "Quanto é que você e o seu cônjuge concordam sobre as metas, objetivos e outras coisas consideradas importantes", $\alpha=.84)$; Satisfação (10 itens, e.g. "Alguma vez se arrependeu de ter casado/de viver junto?", $\alpha=.80$ ); Coesão (5 itens, "Com que frequência você e o seu cônjuge conversam calmamente sobre alguma coisa?", $\alpha=.76$ ); e Expressão do Afeto (4 itens, "Estar demasiado cansado pra ter relações sexuais foi um problema nas últimas semanas?", $\alpha=.68)$. Os itens são sobretudo do tipo Likert (e.g. 1=Concordamos sempre a 6=Discordamos sempre), existindo também dois itens dicotómicos (Sim ou Não).

\section{Bem-estar pessoal}

Usámos a versão portuguesa (Matos, André, Cherpe, Rodrigues, Figueira, \& Marques Pinto, 2010) do Mental Health Continuum - Short Form (MHC-SF; Keyes et al. 2008) para avaliar a saúde mental positiva de acordo com as três dimensões do bem-estar pessoal, variável individual escolhida para verificação da validade de critério. O MHC-SF é a versão breve do questionário e inclui 14 itens que avaliam quanto o indivíduo experienciou, no último mês: (a) a presença de sentimentos positivos e uma avaliação cognitiva de satisfação com a vida em geral (Bem-estar Emocional, 3 itens, e.g. "Interessado pela vida", $\alpha=.84$ ); (b) o funcionamento positivo na vida individual (Bem-estar Psicológico, 6 itens, e.g. "Confiante para pensar ou exprimir as suas próprias ideias e opiniões”, $\alpha=.84$ ); (c) $\mathrm{o}$ funcionamento positivo na vida de comunidade (Bem-estar Social, 5 itens, e.g. "Que tinha alguma coisa importante para contribuir para a sociedade”, $\alpha=.77)$. Os participantes avaliaram a frequência de cada sintoma numa escala tipo Likert de 6 pontos (1=nunca a $6=$ todos os dias). Pontuações mais elevadas em cada escala e no valor global $(\alpha=.88)$ indicam níveis superiores de bem-estar e saúde mental.

\section{Informação sociodemográfica}

Aos participantes era pedido que respondessem a um conjunto de questões mais gerais de âmbito sociodemográfico e relacional, como sejam a idade, nível de educação, ocupação, estatuto relacional, tempo de relação conjugal, etc.

\section{Análises Estatísticas}

O programa SPSS versão 19.0 para o Windows e o software AMOS foram os recursos utilizados para os procedimentos de análise de dados. Utilizámos estatísticas descritivas como frequências, médias e desvios-padrão. Para o estudo de validação do QCCP, adotámos uma abordagem confirmatória para testar a validade fatorial do modelo de medida proposto pelos autores. Considerámos que o modelo se ajustaria aos dados se os valores de CFI, TLI e GFI fossem superiores a .95 e os de RMSEA inferiores a .60 (Hu \& Bentler, 1999), e os de $\chi^{2} / d f$ iguais ou inferiores a 3 (Segars \& Grover, 1993). Valores de RMSEA entre .06 e .08 seriam considerados aceitáveis, entre .08 e .10 toleráveis e inaceitáveis se acima de .10 (Browne \& Cudeck, 1992). O refinamento do modelo baseou-se nos índices de modificação calculados pelo AMOS, que prosseguimos apenas quando considerados adequados do ponto de vista estatístico e teórico. Eliminámos itens e/ou modificámos trajetórias quando os índices de modificação eram superiores 
Quadro 1. Questionário de Coping Conjugal Prospetivo (inicial com oito itens)

\section{QCCP}

(Greenglass, Schwarzer \& Taubert, 1999; versão portuguesa de Alvarez, Marques Pinto e Gregg, 2010; versão adaptada de Costa Ramalho, Marques Pinto \& Ribeiro, 2011)

As frases que se seguem representam reações que as pessoas podem ter face a várias situações no contexto da relação conjugal. Indique quão verdadeiras são estas frases para si, dependendo de como se sente em cada situação. Utilize a chave de classificação que se segue e assinale com uma cruz (X) ou com um círculo (O) o número que escolher.

\begin{tabular}{|c|c|c|c|c|}
\hline & Nunca & Raramente & $\begin{array}{l}\text { Algumas } \\
\text { vezes }\end{array}$ & Sempre \\
\hline $\begin{array}{l}\text { 1. Quando tenho um problema na relação } \\
\text { conjugal tomo a iniciativa de o resolver. }\end{array}$ & 1 & 2 & 3 & 4 \\
\hline $\begin{array}{l}\text { 2. Aperfeiçoo as minhas capacidades } \\
\text { relacionais para prevenir que a relação } \\
\text { conjugal termine ou corra mal. }\end{array}$ & 1 & 2 & 3 & 4 \\
\hline $\begin{array}{l}\text { 3. Sou uma pessoa com iniciativa para } \\
\text { investir/cuidar da minha relação } \\
\text { conjugal. }\end{array}$ & 1 & 2 & 3 & 4 \\
\hline $\begin{array}{l}\text { 4. Previno-me para evitar situações que } \\
\text { possam pôr em perigo a minha relação } \\
\text { conjugal. }\end{array}$ & 1 & 2 & 3 & 4 \\
\hline $\begin{array}{l}\text { 5. Face a uma dificuldade com o meu } \\
\text { cônjuge, espero que as coisas se } \\
\text { resolvam por si próprias. }\end{array}$ & 1 & 2 & 3 & 4 \\
\hline $\begin{array}{l}\text { 6. Procuro garantir que a minha relação } \\
\text { conjugal está segura para a proteger de } \\
\text { dificuldades no futuro. }\end{array}$ & 1 & 2 & 3 & 4 \\
\hline $\begin{array}{l}\text { 7. Tento determinar o que preciso fazer } \\
\text { para que a relação conjugal tenha } \\
\text { sucesso. }\end{array}$ & 1 & 2 & 3 & 4 \\
\hline $\begin{array}{l}\text { 8. Preparo-me para acontecimentos } \\
\text { desfavoráveis na relação conjugal. }\end{array}$ & 1 & 2 & 3 & 4 \\
\hline
\end{tabular}

a $11\left[\chi^{2}(1)=10.86, p=.001\right]$. Usámos os índices Akaike Information Criterion (AIC) e o Expected Cross-Validation Index (ECVI) para comparar a qualidade dos modelos alternativos, considerando os valores mais baixos como indicando melhor ajustamento. Realizámos análises de variância (oneway ANOVA) das médias e desvios padrão do coping conjugal prospetivo em termos das variáveis sócio-demográficas-relacionais que considerámos significativas. $\mathrm{O}$ modelo preditivo foi avaliado com base em análises de regressão múltipla.

\section{Resultados}

\section{Análise fatorial confirmatória}

Com o objetivo de testar o modelo de medida proposto nesta versão adaptada à conjugalidade, procedemos a uma Análise Fatorial Confirmatória do instrumento. Numa primeira análise, a AFC mostrou que o modelo original proposto (4 itens preventivo e 4 itens proactivo) tinha um ajustamento sofrível na presente amostra $\left(\chi^{2} / \mathrm{df}=\right.$ 5.03, GFI=.94, CFI=.87, TLI=.80, RMSEA=.095). Os valores de AIC e ECVI foram respetivamente de 129.49 e .288 . Partiu-se para a observação dos índices de modificação para avaliar de que modo - 
eliminando vias não significativas - o modelo poderia ser ajustado.

De acordo com Stevens (2009), considerando a dimensão da nossa amostra, o critério para inclusão de cada item no fator aponta para um loading superior a .254. Assim, procedeu-se à retirada do item 5 da análise (peso fatorial de .186) e alguns erros de medida dentro do mesmo fator foram correlacionados. Os índices de ajustamento deram sinais da adequação deste segundo modelo à nossa amostra: $\chi^{2} / \mathrm{df}=2.84$, GFI=.98, CFI=.97, TLI=.93, RMSEA=.064. Os valores de AIC e ECVI diminuíram para, respetivamente, 64.36 e .143 reforçando o melhor ajustamento deste modelo.

Avaliando a consistência interna das subescalas assim propostas (retirada do item $5 \mathrm{da}$ subescala coping proactivo), obtivemos: coping proactivo ( 3 itens), $\alpha=.55$; coping preventivo, $\alpha=.57$. Os índices de confiança desta subescala revelaram-se também afetados pelo valor muito baixo da correlação item 8 - total ( $r=.19)$, sendo que o alpha de Cronbach da subescala de coping preventivo aumentou para $.65 \mathrm{com}$ a sua retirada. Optámos assim por retirar mais um item da análise (este da subescala preventiva) (Field, 2013; Nunnally \& Bernstein, 1994; Maroco \& Garcia-Marques, 2006).

Após eliminação dos itens 5 e 8 da análise, procedemos a nova AFC. Todos os índices deste terceiro modelo revelaram ainda melhor ajustamento: $\chi^{2} / \mathrm{df}=1.27, \quad \mathrm{GFI}=.99, \quad \mathrm{CFI}=.99$, TLI=.99, RMSEA=.025. Os valores de AIC e ECVI diminuíram para 37.64 e .084 . Contudo, os valores questionáveis da consistência interna levaram-nos a testar o ajustamento de um modelo unifatorial com os oito itens agrupados.

Com este quarto modelo - unifatorial de oito itens - obtivemos como índices de ajustamento: $\chi^{2} / \mathrm{df}=4.90, \quad \mathrm{GFI}=.94, \quad \mathrm{CFI}=.86, \quad \mathrm{TLI}=.81$, RMSEA $=.094$. Os valores de AIC e ECVI foram respetivamente 130.03 e .289. Com a evidência do modelo não ajustado aos nossos dados, partimos para a observação dos índices de modificação para avaliar de que modo - eliminando vias não significativas de acordo com o critério de loading superior a .254 anteriormente referido. Assim, procedeu-se à retirada do item 5 da análise (peso fatorial de.167) e alguns erros de medida foram correlacionados.

Revista Iberoamericana de Diagnóstico y Evaluación - e Avaliação Psicológica. RIDEP · №42 · Vol.2 $161-176 \cdot 2016$
Os índices de ajustamento deste quinto modelo foram: $\chi^{2} / \mathrm{df}=1.69, \mathrm{GFI}=.99, \mathrm{CFI}=.86$, TLI=.97, RMSEA=.039. Os valores de AIC e ECVI desceram respetivamente para 53.21 e .118. A precisão da medida foi de .70 , valor que se encontra na fronteira da adequação da fiabilidade de um instrumento (Nunnally, 1978). Verificamos que este índice de confiança do questionário se revelava afetado pelo valor muito baixo da correlação item 8 - total ( $r=.21$ ), sendo que o alpha de Cronbach da escala aumentou para .74 com a sua retirada. Optámos assim por retirar também este item da análise (Field, 2013; Nunnally \& Bernstein, 1994; Maroco \& GarciaMarques, 2006) e proceder a nova AFC.

Com este sexto e último modelo, obtivemos como índices de ajustamento: $\chi^{2} / \mathrm{df}=1.26$, $\mathrm{GFI}=.99$, CFI=.99, TLI=.99, RMSEA=.024. Os valores de AIC e ECVI diminuíram ainda para 36.83 e .082. Todos os índices de ajustamento apontaram para o modelo unifatorial com seis itens como o que obteve maior adequação à nossa amostra.

Figura 1. Análise Fatorial Confirmatória do Questionário de Coping Conjugal Prospetivo

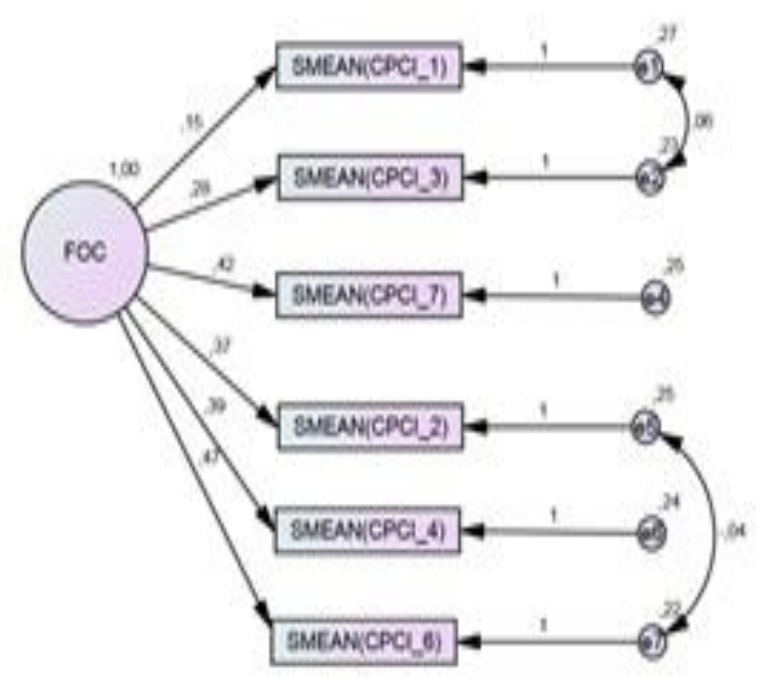

$X 2(7)=8,825 ; p=206 ; X 2 / g l=1,261 ; C F|=, 906 ; P C F|=, 465 ; G F \mid=, 994 ;$ TLI ,992; RMSEA ,024; $P($ rmsea $<10.05)=, 813$

\section{Análise dos itens}

Analisando a sensibilidade da medida, considerou-se que os valores de assimetria superiores a três e os valores de curtose superiores a sete representariam desvios significativos à normalidade. Como pode observar-se no Quadro 
2, encontrámos uma adequada distribuição dos resultados, verificando-se que todos os itens e a escala total apresentaram valores de simetria e ajustamento próximos da distribuição normal.

Para avaliar a discriminação dos itens, as suas médias foram comparadas entre os respondentes que pontuaram abaixo do percentil 25 e aqueles que pontuaram acima do percentil 75 no questionário. O Quadro 3 evidencia que todos os itens têm poder discriminatório significativo ( $p<.001)$, indicando que o Questionário de Coping Conjugal Prospetivo discrimina adequadamente os indivíduos com estilo de coping orientado para o futuro daqueles que evidenciam menos esse estilo.

Quadro 2. Estatísticas descritivas $(\mathrm{N}=451)$, sensibilidade e peso fatorial dos itens do Questionário de Coping Conjugal Prospetivo (final, com seis itens)

\begin{tabular}{lccccc}
\hline & $\begin{array}{c}\text { Peso } \\
\text { Fatorial }\end{array}$ & $M$ & $D P$ & $\begin{array}{c}\text { Assime } \\
\text {-tria }\end{array}$ & $\begin{array}{c}\text { Curto } \\
\text {-se }\end{array}$ \\
\hline Item 1 & .271 & 3.37 & .54 & -.02 & -.93 \\
Item 2 & .598 & 3.29 & .62 & -.64 & 1.04 \\
Item 3 & .496 & 3.48 & .56 & -.45 & -.83 \\
Item 4 & .617 & 3.40 & .63 & -.72 & .34 \\
Item 6 & .710 & 3.40 & .66 & -.92 & .91 \\
Item 7 & .648 & 3.34 & .65 & -.66 & .27 \\
$\begin{array}{l}\text { Resultado } \\
\text { global }\end{array}$ & -- & 3.38 & .40 & -.45 & .02 \\
\hline
\end{tabular}

Diferenças de acordo com variáveis sociodemográficas-relacionais

A realização de testes Oneway ANOVA não evidenciou diferenças significativas no perfil de coping conjugal prospetivo entre mulheres $(M=3.39)$ e homens $(M=3.37)$, nem entre participantes casados $(M=3.40)$ e em união de facto $(M=3.35)$. Não se verificou nenhuma associação entre o nível de escolaridade, a idade ou a duração da relação conjugal e o coping conjugal prospetivo. Os participantes sem filhos evidenciaram níveis significativamente superiores $(M=3.44)$ de coping conjugal prospetivo quando comparados com os que tinham filhos $(M=3.34)$, para $p=.019$. Não se verificaram diferenças nesta dimensão quando comparámos aqueles que se encontravam numa primeira relação conjugal e os restantes.

\section{Validade de critério concorrente \\ Correlações de Pearson entre as dimensões estudadas}

Foram calculadas as correlações de Pearson (Cronbach, 1970; Maroco \& Garcia-Marques, 2006) entre o QCCP e as medidas de autorrelato de ajustamento diádico (EAM) e bem-estar pessoal (MHC-SF). A matriz de correlações é apresentada no Quadro 4. Como esperado, o coping conjugal prospetivo correlacionou-se positivamente com todos os indicadores de bemestar individual e relacional ( $r=.30$ a $r=.49$.), indicando que níveis superiores de coping conjugal prospetivo se relacionaram com índices mais elevados de ajustamento diádico e de bemestar pessoal, em todas as suas dimensões. As dimensões do ajustamento diádico revelam associações positivas moderadas a fortes entre si (de $p=.434$ a $p=.667$ ). Também as diferentes dimensões do bem-estar individual se correlacionam positiva e significativamente (de $p=.405$ a $p=.552$ ), influenciando-se mutuamente mas sem perda do seu contributo diferenciado, como sucedera já em estudos portugueses anteriores (e.g. Figueira, Marques Pinto, Lima, Matos, \& Cherpe, 2014).

\section{Efeito preditivo do Coping Conjugal Prospetivo no Ajustamento Diádico e no Bem-Estar Pessoal}

Realizámos análises de regressão múltipla para avaliar em que medida as estratégias de coping conjugal prospetivo eram um preditor significativo do ajustamento diádico global e das suas quatro dimensões, assim como das três dimensões do bem-estar subjetivo. Os resultados apresentam-se no Quadro 5.

Podemos verificar que o coping conjugal prospetivo explicou uma proporção de variância significativa em todas as dimensões do ajustamento diádico, com $24 \%$ do valor da escala global a ser explicado pelo uso destas estratégias. No que concerne o bem-estar pessoal, a variância explicada manteve-se significativa mas revelou-se inferior, não ultrapassando os $9 \%$. Os valores do beta estandardizados evidenciaram o coping conjugal prospectivo como preditor positivo de todas as dimensões avaliadas. 
Quadro 3. Análise discriminatória dos itens entre os indivíduos que pontuaram alto e baixo no QCCP

\begin{tabular}{cccc}
\hline $\begin{array}{c}\text { Número } \\
\text { do item }\end{array}$ & $\begin{array}{c}\text { Média (DP) do } \\
\text { grupo < percentil 25 }\end{array}$ & $\begin{array}{c}\text { Média (DP) do grupo } \\
>\text { percentil } 75\end{array}$ & Teste $T$ \\
\hline & $(n=169)$ & $(n=89)$ & \\
1 & $3.12(.50)$ & $3.80(.40)$ & $-11.09 * * *$ \\
2 & $2.88(.51)$ & $3.94(.23)$ & $-22.96 * * *$ \\
3 & $3.10(.51)$ & $3.98(.15)$ & $-20.82^{* * *}$ \\
4 & $2.94(.53)$ & $3.92(.27)$ & $-19.91 * * *$ \\
6 & $2.88(.61)$ & $3.92(.27)$ & $-19.02 * * *$ \\
7 & $2.83(.57)$ & $3.92(.27)$ & $-20.93 * * *$ \\
\hline
\end{tabular}

Nota. $* * * p<.001$

\section{Discussão}

Apesar das evidências relativas ao papel do coping orientado para o futuro em diversos índices de bem-estar (e.g. Gan et al., 2007; Greenglass, Fiksenbaum, \& Eaton, 2006), a investigação não se tem focado na compreensão destes processos no âmbito da conjugalidade. Neste contexto, o objetivo central deste estudo foi adaptar e validar o Questionário de Coping Conjugal Prospetivo numa amostra da população portuguesa, explorando também o seu valor preditivo relativamente a duas dimensões positivas, uma individual e outra relacional: o bem-estar pessoal e o ajustamento diádico. Diferentemente de estudos prévios que confirmaram empiricamente uma estrutura bifatorial com a distinção entre o coping proactivo e o coping preventivo, assim como a validade descriminante entre os dois (e.g. Gan et al., 2007), identificámos no nosso estudo uma estrutura de fator único que designámos - como já Gan et al. (2007) tinham feito - de coping prospetivo (ou orientado para o futuro). O QCCP apresenta consistência interna considerada apropriada (Nunnally, 1978) ou aceitável (George, \& Mallery, 2003), boa discriminação dos itens e boa validade concorrente.

Em termos conceptuais, Schwarzer e colaboradores (Schwarzer \& Knoll, 2003; Schwarzer \& Taubert, 2002) tinham notado que muito embora com correlações interfator menores que .55 - as dimensões proativa e preventiva se manifestam muitas vezes no mesmo tipo de comportamentos (e.g. desenvolvimento de competências, acumulação de recursos)
Por este motivo, referem que "não é imediatamente claro se um determinado comportamento deve ser considerado preventivo ou proactivo" (Schwarzer \& Knoll, 2003, p. 402) e antecipam potenciais redundâncias nos dois construtos. A ausência de validade discriminante entre os dois tipos de coping revelou-se um dado evidente no nosso estudo pelo que viemos a assumir que, no contexto das relações conjugais, a preparação dos indivíduos para potenciais stressores se (con)funde com os esforços para alcançar objetivos e promover o crescimento pessoal. Ficou evidente a validade de critério da variável coping conjugal prospetivo, encontrandose correlações moderadas entre esta variável e os diversos índices de bem-estar individual e conjugal avaliados. Evidencia-se o poder preditivo do coping conjugal prospetivo relativamente ao bem-estar individual e à dimensão relacional do ajustamento diádico.

Os dados que apontam para níveis significativamente superiores de coping conjugal prospetivo nos participantes sem filhos, quando comparados com aqueles com filhos, devem ser considerados preliminares abrindo pistas para investigações futuras. Considerando que não encontramos estudos publicados sobre o papel do coping prospetivo no contexto das relações conjugais, poderemos apenas hipotetizar sobre a maior acumulação de recursos que promovam o crescimento pessoal e a consecução de objetivos (características do coping prospetivo) no contexto de uma relação de casal sem filhos. Quando aos papéis e tarefas conjugais se somam os papéis parentais - nomeadamente nos primeiros anos de vida dos filhos, como é o caso da nossa amostra - 
Quadro 4. Médias, desvio-padrão, correlações de Pearson e consistência interna das variáveis em estudo $(\mathrm{N}=451)$

\begin{tabular}{cccccc}
\hline Variáveis & & $M$ & $S D$ & Pearson & $\alpha$ \\
\hline $\begin{array}{c}\text { Coping conjugal } \\
\text { prospetivo }\end{array}$ & & $3.38(1-4)$ & .40 & & \\
\hline Ajustamento & & $4.59(1-6)$ & .46 & $.49^{* *}$ & .91 \\
& Consenso & 4.82 & .53 & $.42^{* *}$ & .84 \\
& Satisfação & 4.91 & .53 & $.44^{* *}$ & .80 \\
& Coesão & 4.42 & .74 & $.39^{* *}$ & .76 \\
& Exp Afeto & 3.26 & .51 & $.30^{* *}$ & .68 \\
\hline BE emocional & & $4.88(1-6)$ & .83 & $.31^{* *}$ & .84 \\
BE social & & $3.14(1-6)$ & 1.00 & $.21^{* *}$ & .77 \\
BE psicológico & & $4.32(1-6)$ & .92 & $.30^{* *}$ & .84 \\
\hline
\end{tabular}

Nota. ** Correlação significativa para $p<.01$

é possível que, por um lado, a fadiga, a sobrecarga, e o stress associado às tarefas parentais diminuam a proatividade dos cônjuges na atenção à relação de casal (e.g. Trillingsgaard, Baucom, Heyman, \& Elklit, 2012); por outro lado, os objetivos e os esforços de desenvolvimento tenderão a ser menos centrados no casal e mais centrados na prole (e.g. Biehle, 2009).

Os nossos dados corroboram estudos anteriores (e.g. Wilson et al., 2005) que evidenciaram - noutros contextos que não a relação conjugal - a associação positiva entre a autorregulação relacional e a satisfação relacional, com aquela a explicar entre um quarto a um terço da variância na satisfação quer em relações conjugais recentes quer de longa duração. Halford et al. (2007) alargaram estes resultados, tendo colocado em evidência que a autorregulação relacional se associa concorrente e prospectivamente (e com grande magnitude) com o intercepto da satisfação relacional. Para além disso, esta associação prospetiva surge como unidirecional e não pode ser explicada por a satisfação relacional causar relatos inferiores de autorregulação. Também Greenglass et al. (2006) enfatizam que o coping proactivo se associa com um melhor funcionamento psicológico em diversas medidas. Encontraram uma associação do coping proactivo com níveis mais baixos de depressão, tal como sucedera nos estudos prévios de adaptação da escala PCI (e.g. Greenglass, 2006; Pasikowski et al., 2002). O coping proactivo tem-se revelado claramente como um estilo de coping ativo baseado na iniciativa individual, no otimismo e na autodeterminação
(Greenglass et al., 2006) e na mobilização de recursos (Greenglass et al., 1999), o que parece torná-lo inconsistente com a depressão, o evitamento e comportamentos autodestrutivos.

Parece pois que este compósito atitudinal e comportamental do coping que se orienta ativamente para o futuro da relação conjugal, ora construindo recursos que facilitem ultrapassar desafios, alcançar objetivos e promover o desenvolvimento pessoal, ora prevenindo a ocorrência ou o dano causado por potenciais stressores, tem um papel relevante no contexto da conjugalidade. De acordo com a definição de Schwarzer e Taubert (2002), esta dimensão pode possibilitar que os indivíduos percebam as situações mais exigentes (neste caso, da relação conjugal) como desafios que importa ultrapassar. Os dados presentes evidenciam que, por oposição à inatividade, o foco individual no potencial de mudança e na construção de recursos pessoais tem consequências benéficas também ao nível das relações conjugais.

Em síntese, este estudo alargou o âmbito do coping prospetivo enquanto conceito da psicologia positiva para o âmbito das relações de casal. Para lá de outros benefícios previamente encontrados - e.g. na procura de emprego, $\mathrm{Hu} \&$ Gan, 2011; no ajustamento à universidade, Gan et al., 2007 - esta tendência para agir à frente do tempo revela-se preditora de importantes dimensões individuais (bem-estar subjetivo) e relacionais (ajustamento diádico). Em linha com estudos prévios sobre os mecanismos de autorregulação relacional (Halford et al., 2007; Weigel \& Ballard-Reisch, 1999), os nossos dados 
Quadro 5. Síntese das análises de regressão múltipla para o coping conjugal prospetivo como preditor das dimensões de bem-estar pessoal e ajustamento diádico $(\mathrm{N}=450)$

\begin{tabular}{|c|c|c|c|c|}
\hline Variável & $b$ & $S E b$ & $\mathrm{~B}$ & $p$ \\
\hline \multicolumn{2}{|c|}{ Ajustamento Diádico - Global } & \multicolumn{3}{|c|}{$R^{2}=.24 ; F(1,449)=144.27, p<.001$} \\
\hline Constante & 2.67 & .16 & & $<.001$ \\
\hline MPCI & .57 & .05 & .49 & $<.001$ \\
\hline \multicolumn{2}{|c|}{ Ajustamento Diádico - Consenso } & \multicolumn{3}{|c|}{$R^{2}=.17 ; F(1,449)=96.78, p<.001$} \\
\hline Constante & 2.93 & .19 & & $<.001$ \\
\hline MPCI & .56 & .06 & .42 & $<.001$ \\
\hline \multicolumn{2}{|c|}{ Ajustamento Diádico - Satisfação } & \multicolumn{3}{|c|}{$R^{2}=.19 ; F(1,449)=105.49, p<.001$} \\
\hline Constante & 2.96 & .19 & & $<.001$ \\
\hline MPCI & .58 & .06 & .44 & $<.001$ \\
\hline \multicolumn{2}{|c|}{ Ajustamento Diádico - Coesão } & \multicolumn{3}{|c|}{$R^{2}=.15 ; F(1,449)=80.66, p<.001$} \\
\hline Constante & 2.01 & .27 & & $<.001$ \\
\hline MPCI & .71 & .08 & .39 & $<.001$ \\
\hline \multicolumn{2}{|c|}{ Ajustamento Diádico - Exp Afeto } & \multicolumn{3}{|c|}{$R^{2}=.09 ; F(1,449)=45.62, p<.001$} \\
\hline Constante & 1.95 & .20 & & $<.001$ \\
\hline MPCI & .39 & .06 & .30 & $<.001$ \\
\hline \multicolumn{2}{|c|}{ Bem-estar emocional } & \multicolumn{3}{|c|}{$R^{2}=.09 ; F(1,449)=46.27, p<.001$} \\
\hline Constante & 2.74 & .32 & & $<.001$ \\
\hline \multirow{2}{*}{\multicolumn{2}{|c|}{$\begin{array}{r}.63 \\
\text { Bem-estar social }\end{array}$}} & .09 & .31 & $<.001$ \\
\hline & & \multicolumn{3}{|c|}{$R^{2}=.04 ; F(1,449)=20.59, p<.001$} \\
\hline Constante & 1.37 & .39 & & .001 \\
\hline \multirow{2}{*}{\multicolumn{2}{|c|}{ Bem-estar psicológico }} & .12 & .21 & $<.001$ \\
\hline & & \multicolumn{3}{|c|}{$R^{2}=.09 ; F(1,449)=44.07, p<.001$} \\
\hline Constante & 2.00 & .35 & & $<.001$ \\
\hline MPCI & .69 & .10 & .30 & $<.001$ \\
\hline
\end{tabular}

vêm reforçar que a sua maior utilização pelos parceiros amorosos se associa a resultados individuais e conjugais mais positivos. No entender de Baumeister e Stillman (2008), “a conexão entre a autorregulação e as relações íntimas é recíproca: relações íntimas saudáveis não apenas realizam boa autorregulação, elas na verdade fortalecem a autorregulação" (p.1)

Este estudo tem diversas implicações práticas, disponibilizando evidência empírica atual para compreensão dos perfis de coping de indivíduos adultos no contexto das suas relações de casal, assim como do seu impacto no bem-estar pessoal e no ajustamento diádico. Para aqueles que optam - no seu projeto de vida - pela vivência de uma relação conjugal, a perceção de bem-estar individual e relacional constitui um objetivo primário. Para muitos, porém, a aventura conjugal não se afigura fácil ou mesmo possível de manter (Morris \& Carter, 2009), observando-se nos últimos anos - nomeadamente em Portugal - um número crescente e cada vez mais precoce de separações e divórcios (INE, 2013). Um fator sem dúvida importante será a dificuldade em lidar com as exigências/desafios de uma vida a dois. Este estudo suporta o interesse em integrar a dimensão do coping prospetivo na terapia e aconselhamento conjugal, assim como em programas de educação ou enriquecimento conjugal que tenham como objetivo potenciar resultados de bem-estar. Será importante consciencializar e refletir com os indivíduos esta perspetiva de se assumir um papel ativo na construção e manutenção da relação conjugal, sabendo que os stressores $\mathrm{e}$ as exigências são parte integrante de qualquer vivência relacional. Em estudos prévios, Baumeister, Gailliot, DeWall, e Oaten (2006) enfatizam que a autorregulação pode ser fortalecida através da prática. Também Meraz e Palencia (2012) sublinham a importância das estratégias de manutenção da relação de casal: muito embora estas não garantam o sucesso por si mesmas, e ainda que se saiba hoje que os resultados relacionais são condicionados por 
diferentes variáveis e circunstâncias, os casais que mais as colocam em prática terão de certo maior probabilidade de construir uma relação satisfatória.

De acordo com os resultados de Sohl e Moyer (2009), as intervenções que pretendam fortalecer os benefícios do coping proativo devem focar-se sobretudo na promoção de recursos e na definição realista de objetivos. Alterar estas competências parece mais eficaz do que agir diretamente sobre o coping proativo, podendo eventualmente levar ao mesmo resultado. Nos últimos anos, foram já desenvolvidas intervenções para promoção das competências proativas no envelhecimento (Bode, Ridder, Kuijer, \& Bensing, 2007) e para promover a personalidade proativa no desempenho académico (Kirby, Kirby, \& Lewis, 2002). Existem, segundo os autores, muitos outros contextos a ser explorados, como é o caso do sucesso nas relações conjugais, podendo beneficiar amplamente de intervenções semelhantes (Sohl \& Moyer, 2009).

O presente estudo constitui um importante contributo empírico relativo à pertinência do coping prospetivo no âmbito da conjugalidade. Muito embora o QCCP apresente boas propriedades psicométricas, não encontrámos uma estrutura com dois fatores mas sim unifatorial, o que impossibilita a distinção entre as duas formas de coping, proactivo e preventivo, previstas no modelo de partida. Duas limitações desta investigação residem na sua metodologia, particularmente o facto de todos os construtos serem avaliados apenas com base em questionários de autorrelato num desenho transversal. Por este motivo, não é possível retirar inferências sobre relações causais entre as variáveis. Constituindo o coping um processo que se desenrola ao longo do tempo, que se modifica com avanços e retrocessos - dependendo de fatores individuais e situacionais, desenhos metodológicos longitudinais teriam um contributo a dar para a compreensão de como o coping prospetivo concorre para os processos de crescimento pessoal e de bem-estar individual e relacional no contexto específico das relações conjugais.

A especificidade da amostra (indivíduos portugueses, etnia caucasiana, numa relação conjugal há menos de 11 anos) sugere que seja Revista Iberoamericana de Diagnóstico y Evaluación - e Avaliação Psicológica. RIDEP · No42 · Vol.2 · 161-176 · 2016 usado com cautela em outras amostras e que os seus resultados sejam sempre lidos com precaução. Devem conduzir-se novos estudos que permitam generalizar os resultados para outras culturas. Uma outra limitação prende-se com o facto de este estudo se basear em medidas de autorrelato de indivíduos e não de casais. Por este motivo, não é possível por exemplo avaliar em que medida estratégias de coping prospetivo se associam positivamente com as perceções sobre o bem-estar pessoal e o ajustamento apenas no caso do próprio ou também no caso do parceiro, o que seria interessante de compreender.

\section{Referências}

Aspinwall, L.G. (2005). The psychology of future-oriented thinking: From achievement to proactive coping, adaptation, and aging. Motivation and Emotion, 29(4), 203-235. doi:10.1007/s11031-006-9013-1.

Bandura, A. (2001). Social cognitive theory: An agentic perspective. Annual Review of Psychology, 52, 1-26. doi:10.1146/annurev.psych.52.1.1.

Baumeister, R. F., Gailliot, M., DeWall, C. N., \& Oaten, M. (2006). Self-regulation and personality: How interventions increase regulatory success, and how depletion moderates the effects of traits on behavior. Journal of Personality, 74(6), 1773-1801. doi:10.1111/j.1467-6494.2006.00428.x.

Baumeister, R. F., \& Stillman, T. F. (2008). Selfregulation and close relationships. In J. V. Wood, A. Tesser, \& J. G. Holmes (Eds.), The self and social relationships (pp. 139-158). New York, NY: Psychology Press.

Biehle, S. N. (2009). Preparing for Parenthood: Individual and Couple Models of Anxiety and Marital Satisfaction (Doctoral dissertation, Kent State University).

Bode, C., de Ridder, D. T. D., Kuijer, R. G., \& Bensing, J. M. (2007). Effects of an intervention promoting proactive coping competencies in middle and late adulthood. The Gerontologist, 47, 42-51. doi:/10.1093/geront/47.1.42.

Browne, M. W., \& Cudeck, R. (1992). Alternative ways of assessing model fit. Sociological Methods and 
Research

21 ,

230-258. doi:10.1177/0049124192021002005.

Cronbach, L.J. (1970). Essentials of psychological testing ( $3^{\text {rd }}$ ed.). New York: Harper \& Row.

Endler, N. S., \& Parker, J. D. A. (1994). Assessment of multidimensional coping: Task emotion, and avoidance strategies. Psychological Assessment, 6, 50-60. doi:10.1037/1040-3590.6.1.50.

Field, A. (2013). Discovering Statistics Using SPSS (4 ${ }^{\text {th }}$ Ed.). London, UK: SAGE Publications Ltd.

Figueira, C., Marques Pinto, A., Lima, L., Matos, A.P., \& Cherpe, S. (2014). Adaptação do Mental Health Continuum - LF - for adults em estudantes universitários portugueses. Revista Iberoamericana de Diagnóstico y Evaluación - e Avaliação Psicológica, 38(2), 93-116.

Fiksenbaum, L. Greenglass, E.R. \& Eaton, J. (2001). Proactive coping, wellbeing and adjustment in the elderly. Paper presented at the $22^{\text {nd }}$ Meeting of the Stress and Anxiety Research Society (STAR), Palma de Mallorca.

Gan, Y., Yang, M., Zhou, Y., \& Zhang, Y. (2007). The two-factor structure of future-oriented coping and its mediating role in student engagement. Personality and Individual Differences, $\quad 43, \quad 851-863$. doi:10.1016/j.paid.2007.02.009.

George, D., \& Mallery, P. (2003). SPSS for Windows step by step: A simple guide and reference. 11.0 update (4th ed.). Boston: Allyn \& Bacon.

Greenglass, E. R. (2002a). Proactive coping. In E. Frydenberg (Ed.), Beyond coping: Meeting goals, vision, and challenges (pp. 37-62). London: Oxford University Press.

Greenglass, E. (2002b). Proactive coping, stress, and social support: Psychological implications. Paper presented at the XXV International Congress of Applied Psychology, Singapore.

Greenglass, E.R. (2005). Proactive coping, resources and burnout: Implications for occupational stress. In A.S.G. Antonious, \& C. Cooper (Eds.), Research companion to organizational health psychology (pp. 503-515). London: Edward Elgar.
Greenglass, E.R. (2006). Vitality and vigor: Implications for healthy functioning. In $\mathrm{P}$. Buchwald (Ed.), Stress and anxiety - Application to health, community, work place and education (pp. 65-86). Cambridge, UK: Scholars Press.

Greenglass, E.R., Fiksenbaum, L., \& Eaton, J. (2006). The relationship between coping, social support, functional disability and depression in the elderly. Anxiety, Stress, and Coping, 19(1), 15-31. doi:10.1080/14659890500436430

Greenglass, E.R., Schwarzer, R., Jakubiec, D., Fiksenbaum, L., \& Taubert, S. (1999). The Proactive Coping Inventory (PCI): A Multidimensional Research Instrument. Paper presented at the 20th International Conference of the Stress and Anxiety Research Society (STAR), Cracow, Poland, July 12-14.

Greenglass, E.R., Stokes, P., \& Fiksenbaum, L. (2005). Engagement, work stress and wellbeing: Implications for psychological functioning. The Journal of the International Stress Management Association, 17, 4-7.

Halford, W. K., Lizzio, A., Wilson, K. L., \& Occhipinti, S. (2007). Does working at your marriage help? Couple relationship selfregulation and satisfaction in the first 4 years of marriage. Journal of Family Psychology, 21, 185-194. doi:10.1037/08933200.21.2.185.

Halford, W. K., Wilson, K.L., Lizzio, A., \& Moore, E. (2002). Does working at a relationship work? Relationship self-regulation and relationship outcomes. In P. Noller \& J.A. feeney (Eds.), Understanding marriage: Developments in the study of couple interaction (pp. 493-517). Cambridge: Cambridge University Press.

Halford, W. K., Sanders, M. R., \& Behrens, B. C. (1994). Self-regulation in behavioral couples' therapy. Behavior Therapy, 25, 431-452. doi:10.1016/S0005-7894(05)80156-X.

Herdman, M., Fox-Rushby, J., \& Badia, X. (1998). A model of equivalence in the cultural adaptation of HRQoL instruments: The universalist approach. Quality of Life Research, 7, 323-35.

Hu, L., \& Bentler, P.M. (1999). Cutoff criteria for fit indexes in covariance structure analysis: Conventional criteria versus new alternatives. Structural Equation Modeling: A 
Multidisciplinary Journal, 6(1), 1-55. doi:10.1080/10705519909540118.

Hu, Y., \& Gan, Y. (2011). Future-oriented coping and job hunting among college students. The Psychological Record, 61, 253-268.

Instituto Nacional de Estatística (2015). Estatísticas Demográficas 2013. In https://www.ine.pt/. Lisboa: INE Portugal.

Keyes, C.L.M., Wissing, M., Potgieter, J.P., Temane, M., Kruger, A., \& van Rooy, S. (2008). Evaluation of the mental health continuum-short form (MHC-SF) in Setswana-speaking South Africans. Clinical Psychology \& Psychotherapy, 15(3), 181-192. doi: 10.1002/cpp.572.

Kirby, E. G., Kirby, S. L., \& Lewis, M. A. (2002). A study of the effectiveness of training proactive thinking. Journal of Applied Social Psychology, 32, 1538-1549.

Levine, J. R., \& Markman, H. J. (Eds.). (2001). Why do fools fall in love? San Francisco: Jossey-Bass.

Markman, H. J., Stanley, S. M., \& Blumberg, S. L. (2010). Fighting for your marriage: Positive steps for preventing divorce and preserving a lasting love. $\left(3^{\text {rd }} \mathrm{ed}\right.$.). New York, NY: Jossey-Bass.

Maroco, J., \& Garcia-Marques, T. (2006). Qual a fiabilidade do alfa de Cronbach? Questões antigas e soluções modernas? Laboratório de Psicologia, 4, 65-90.

Matos, A.P., André, R. S., Cherpe, S., Rodrigues D., Figueira, C., \& Marques Pinto, A. (2010). Estudo psicométrico preliminar da Mental Health Continuum - Short Form - for youth, numa amostra de adolescentes portugueses. Psychologica, 53, 131-156.

Meraz, M.G., \& Palencia, A.R. (2012). Mantenimiento en la relació de pareja: Construcción y validación de dos escalas. Revista Iberoamericana de Diagnóstico y Evaluación - e Avaliação Psicológica, 34(1), 133-155.

Morris, M.L., \& Carter, S.A. (1999). Transition to Marriage: A Literature Review. Journal of Family and Consumer Sciences Education, 17(1), 1-21.

Nunnally, J. C. (1978). Psychometric theory $\left(2^{\text {nd }}\right.$ ed.). New York: McGraw-Hill.
Nunnally, J.C., \& Bernstein, I.H. (1994). Psychometric Theory. USA: McGraw-Hill, Inc.

Pasikowski, T., Sek, H., Greenglass, E., \& Taubert, S. (2002). The Proactive Coping Inventory - Polish adaptation. Polish Psychological Bulletin, 33, 4146.

Relvas, A.P. (1996). O ciclo vital da família: Perspectiva sistémica. Porto: Edições Afrontamento.

Schwarzer, R. (1999). Proactive Coping Theory. Paper presented at the $20^{\text {th }}$ International Conference of the Stress and Anxiety Research Society (STAR), Cracow, Poland, July 12-14, 1999.

Schwarzer, R. (2000). Manage stress at work through preventive and proactive coping. In $\mathrm{E}$. A. Locke (Ed.), The Blackwell handbook of principles of organizational behavior (pp. 342-355). Oxford, UK: Blackwell.

Schwarzer, R., \& Knoll, N. (2003). Positive coping: Mastering demands and searching for meaning. In S.J. Lopez \& C.R. Snyder (Eds.), Handbook of positive psychological assessment (pp. 393-409). Washington, DC: American Psychological Association.

Schwarzer, R., \& Taubert, S. (2002). Tenacious goal pursuits and striving toward personal growth: Proactive coping. In E. Frydenberg (Ed.), Beyond coping: Meeting goals, visions and challenge (pp. 19-35). London: Oxford University Press.

Segars, A. H., \& Grover, V. (1993). Re-examining perceived ease of use and usefulness: A Confirmatory Factor Analysis. MIS Quarterly, 17, 517-525. doi: 10.2307/249590.

Seligman, M. E. P. (1990). Learned optimism. New York: Simon \& Schuster.

Shafer, K., Jensen, T. M., \& Larson, J. H. (2012). Relationship effort, satisfaction, and stability across union type. Journal of Marital \& Family Therapy. Advance online publication: doi: 10.1111/jmft.12007.

Sohl, S.J., \& Moyer, A. (2009). Refining the conceptualization of a future-oriented selfregulatory behavior: Proactive coping. Personality and Individual Differences, 47, 139-144. doi:10.1016/j.paid.2009.02.013.

Spanier, G.B. (1976). Measuring dyadic adjustment: New scales for assessing the quality of marriage and similar dyads. Journal 
of Marriage and Family, 38(1), 15-28. doi:10.2307/350547.

Stevens, J. (2009). Applied Multivariate Statistics for the Social Sciences (5th ed.). NY, USA: Routledge.

Trillingsgaard, T., Baucom, K. J., Heyman, R. E., \& Elklit, A. (2012). Relationship interventions during the transition to parenthood: Issues of timing and efficacy. Family Relations, 61(5), 770-783. doi: $10.1111 / \mathrm{j} .174137-$ 29.2012.00730.x

Uskul, A.K., \& Greenglass, E. (2005). Psychological wellbeing in a TurkishCanadian sample. Anxiety, Stress, and Coping, 18(3), 269-278. doi: 10.1080/10615800500205983.

Weigel, D. J., \& Ballard-Reisch, D. S. (1999). Using paired data to test models of relational maintenance and marital quality. Journal of Social and Personal Relationships, 16(2), 175-191. doi:10.1177/0265407599162003. 